\title{
Metabolic parameters of sequential 18F-FDG PET/CT predict overall survival of esophageal cancer patients treated with (chemo-) radiation
}

\author{
Yimin $\mathrm{Li}^{1 \dagger}$, Sebastian Zschaeck ${ }^{2 \dagger}$, Qin Lin ${ }^{1 *}$, Sijia Chen ${ }^{1}$, Lili Chen ${ }^{1}$ and Hua Wu ${ }^{3 *}$
}

\begin{abstract}
Background: To evaluate the prognostic value of metabolic parameters of pre-treatment and interim 18Ffluorodeoxyglucose (FDG) positron emission tomography (PET) for overall survival (OS) of esophageal cancer(EC) patients undergoing (chemo-) radiotherapy.

Methods: A retrospective analysis of 134 patients with pathology confirmed squamous cell EC treated between July 2009 and October 2013 in our hospital was performed. Inclusion criteria for this study were curative intended radiotherapy and availability of pre-treatment and interim 18F-FDG PET. 18F-FDG PET/CT scans were acquired before treatment and after 40 Gray (Gy) of radiotherapy. Maximum standard uptake value (SUV max), metabolic tumor volume(MTV), total lesion glycolysis (TLG), and the percentual changes during both PET scans were recorded. The parameters were named as SUVmax1,MTV1,TLG1,SUVmax2,MTV2,TLG2, $\triangle$ SUV max $_{1} \Delta$ MTV and $\triangle T L G$. The receiver operating characteristic curve (ROC) was used to analyze the relationship between metabolic parameters and OS, survival analysis was carried out by Kaplan-Meier and Cox regression analysis.

Results: Univariate survival analysis showed that SUVmax2、MTV1、 $\mathrm{MTV} 、 T L G 1 、 T L G 2$ and $\triangle T L G$ were associated with OS. Based on the largest Youden index of ROC curves, patients with SUVmax2 $<7.8$, MTV1 $<10.5, \Delta \mathrm{MTV}<0.075$, TLG1 $<59.8, T L G 2<44.3$ and $\triangle T L G<0.27$ tended to live longer. Stratified for these parameters, the estimated median survival time were 27.9 months (m) vs $9.8 \mathrm{~m}, 36.9 \mathrm{~m}$ vs $11.3 \mathrm{~m}, 41.6 \mathrm{~m}$ vs $12 \mathrm{~m}, 48.9 \mathrm{~m}$ vs $14.3 \mathrm{~m}, 32.6 \mathrm{~m}$ vs $13.2 \mathrm{~m}$, and $41.6 \mathrm{~m}$ vs $14.5 \mathrm{~m}$. Cox multi-factor regression analyses revealed SUV $\max 2$ as an independent prognostic factor for OS complementary to TNM staging and the length of primary tumor.
\end{abstract}

Conclusions: Sequential 18F-FDG PET/CT metabolic parameters bear important prognostic value for OS of EC patients. 18F-FDG PET/CT scan before treatment and during chemoradiotherapy seems helpful to evaluate the effect of chemoradiotherapy, guide clinical decisions and provide patients with personalized treatment.

Keywords: Esophageal cancer, 18F-FDG PET/CT, Chemoradiotherapy

\footnotetext{
*Correspondence: linqin05@163.com; wuhua1025@163.com

${ }^{\dagger}$ Yimin Li and Sebastian Zschaeck contributed equally to this work.

'Department of Radiation Oncology, Xiamen Cancer Hospital, the First

Affiliated Hospital of Xiamen University, Xiamen, China

${ }^{3}$ Department of Nuclear Medicine, Xiamen Cancer Hospital, the First Affiliated

Hospital of Xiamen University/Southern Fujian PET Center, Xiamen, China

Full list of author information is available at the end of the article
}

(c) The Author(s). 2019 Open Access This article is distributed under the terms of the Creative Commons Attribution 4.0 International License (http://creativecommons.org/licenses/by/4.0/), which permits unrestricted use, distribution, and reproduction in any medium, provided you give appropriate credit to the original author(s) and the source, provide a link to the Creative Commons license, and indicate if changes were made. The Creative Commons Public Domain Dedication waiver (http://creativecommons.org/publicdomain/zero/1.0/) applies to the data made available in this article, unless otherwise stated. 


\section{Background}

Esophageal cancer (EC) is the eighth most common cancer worldwide with the sixth highest mortality [1]. In China, EC incidence is high, accounting for $50 \%$ of all new global EC cases annually and most of them present with advanced stage. Patients with early stage EC can be treated by surgery alone, while those with locally advanced EC usually receive radiotherapy combined with platinumbased chemotherapy [2]. Neo-adjuvant chemoradiotherapy before surgery is also a common treatment regime $[3,4]$. The optimal primary treatment method is controversial. Both definitive radiochemotherapy (RCT) and preoperative RCT followed by radical surgery are comparable regarding overall survival (OS) of patients; however, conclusive clinical data from state of the art phase III studies are lacking. Moreover, some recent analyses suggest better OS after trimodality treatment [5-8]. Since local recurrences appear to occur more frequently after definitive RCT, trimodality treatment is usually the treatment of choice in medically fit patients; however, in some patients, the location (mostly cervical) or extent of the primary tumour impedes radical tumour excision, or the patient refuses to undergo surgery.

At present, EC treatment outcomes are usually evaluated by computed tomography (CT) and a barium swallow test, but these examinations provide only anatomical information and very coarse functional information. 18F-FDG $\mathrm{PET} / \mathrm{CT}$ is a functional imaging modality that can predict EC prognosis more accurately than conventional CT $[9,10]$.As biological status of tumors is evolving and metabolic tumor response can be detected earlier than morphological tumor response by CT, sequential 18F-FDG PET/ CT seems to be an especially useful prognostic/predictive tool. Some previous studies showed that sequential 18FFDG PET/CT during treatment is able to predict outcomes after radiotherapy and chemotherapy for EC [11].The commonly used metabolic parameters for predicting EC patients' survival include maximum of standard uptake value $\left(\mathrm{SUV}_{\max }\right)$ and metabolic tumor volume (MTV).The prognostic value of $\mathrm{SUV}_{\max }$ remains inconclusive [12-15], and the prognostic value of MTV is affected by esophageal radiation inflammation during radiotherapy [16-18].Total lesion glycolysis (TLG),the product of the MTV and mean SUV, has emerged as a relatively new 18F-FDG PET/CT prognostic parameter in recent years. Studies have shown that TLG is more reliable than MTV because TLG reflects both mean metabolic FDG uptake and tumor volume. Currently, TLG was suggested to predict the treatment outcome of neoadjuvant therapy in smaller number of patients treated in other countries [19, 20], only few studies have been conducted in China to investigate the value of TLG in predicting(chemo-)radiation outcomes for EC.

In our study, 18F-FDG PET/CT was performed before treatment and during radiotherapy in patients with esophageal squamous cell carcinoma undergoing (chemo-)radiation. The sequential 18F-FDG PET/CT metabolic parameters were collected. The aim of our study is to find out whether the metabolic parameters and the change rate of them have prognostic value in Chinese patients.

\section{Methods \\ General information}

We retrospectively analyzed clinical and PET data from EC patients who received radiotherapy and underwent 18F-FDG PET/CT before and during treatment at our hospital between July 2009 and October 2013. Some of the patient data has already been published with focus on other parameters, this is an additional analysis with long-term follow up and the following inclusion criteria: pathology proven esophageal cancer, age $>18$ years, I-IVa disease at staging, initial treatment of esophageal cancer, absence of distant metastasis or other concomitant tumors, no contraindication to radiotherapy or chemotherapy; ECOG score: $0 \sim 2$, Karnofsky performance status(KPS) score $\geq 70$; no major organ dysfunction, no serious anomalies of blood routine test, liver function, pulmonary function, renal function and cardiac function; assay indexes requirement: $\mathrm{WBC} \geq 4.0 \times 109 / \mathrm{L}, \mathrm{ANC} \geq 1.5 \times 109 / \mathrm{L}, \mathrm{PLT} \geq 100 \times 109$ $/ \mathrm{L}, \mathrm{Hb} \geq 90 \mathrm{~g} / \mathrm{L}$; serum bilirubin $\leq 1.5$ times of normal high limit, AST, ALT $\leq 1.5$ times of normal high limit, creatinine $\leq$ normal high limit, normal ECG; 18F-FDG PET/CT before treatment and interim PET at 4-5 weeks during radiotherapy with complete imaging data available.

Clinical staging was determined according to the Non-Surgical Treatment of Esophageal Cancer Clinical Staging Criteria (draft) from the Fifth National Symposium on Radiotherapy for Esophageal Cancer(2010) [21]. Each patient provided written informed consent. A total of 134 EC patients were enrolled, and the clinical features are shown in Table 1.

\section{Radiotherapy}

Patients were treated with conventional fractionated RCT with a single dose of 1.8 or 2 Gy per fraction. From high-resolution contrast-enhanced CT and 18F-FDG PET, gross tumour volume (GTV) was contoured separately for the primary tumour and affected lymph nodes. The clinical target volume (CTV) of the primary tumour was generated by enlarging the primary GTV by $4 \mathrm{~cm}$ in craniocaudal extension of esophageal lesion and by $0.5 \mathrm{~cm}$ in radial extension. Additionally, regional lymph node regions were included with sufficient safety margins within the nodal CTV, and all CTVs were adapted to anatomical structures (excluding bones, lungs or large vessels). The planning treatment volume (PTV) comprised the CTV with additional margins of $0.5 \mathrm{~cm}$. After administration of $50 \mathrm{~Gy}$, an additional radiation boost of 4-16 Gy (average $58.9 \mathrm{~Gy}$ ) was prescribed to a reduced treatment volume 
Table 1 Patient characteristics

\begin{tabular}{|c|c|}
\hline Clinical Features & n (\%) \\
\hline \multicolumn{2}{|l|}{ Age (y) } \\
\hline $62.4 \pm 9$ & - \\
\hline Range (42-88) & - \\
\hline \multicolumn{2}{|l|}{ Sex } \\
\hline $\mathrm{F}$ & $32(23.9 \%)$ \\
\hline M & $102(76.1 \%)$ \\
\hline \multicolumn{2}{|l|}{ Grade of differentiation } \\
\hline Highly differentiated (G1) & $12(9.0 \%)$ \\
\hline Moderately differentiated(G2) & 95 (70.9\%) \\
\hline Poorly differentiated(G3) & $22(16.4 \%)$ \\
\hline Undifferentiated (G4) & $5(3.7 \%)$ \\
\hline \multicolumn{2}{|l|}{ Primary tumor site } \\
\hline Cervical & $13(9.7 \%)$ \\
\hline Upper thorax & $44(32.8 \%)$ \\
\hline Middle thorax & $60(44.8 \%)$ \\
\hline Lower thorax & $14(10.4 \%)$ \\
\hline Mixed & $3(2.2 \%)$ \\
\hline \multicolumn{2}{|l|}{ Length of primary tumor } \\
\hline$<3.5$ & $27(20.2 \%)$ \\
\hline$>3.5$ & $104(77.6 \%)$ \\
\hline Not available & $3(2.2 \%)$ \\
\hline \multicolumn{2}{|l|}{ *T stage } \\
\hline T1 & $3(2.2 \%)$ \\
\hline $\mathrm{T} 2$ & $8(6.0 \%)$ \\
\hline T3 & $43(32.1 \%)$ \\
\hline T4 & $80(59.7 \%)$ \\
\hline \multicolumn{2}{|l|}{${ }^{*} \mathrm{~N}$ stage } \\
\hline NO & $49(36.6 \%)$ \\
\hline N1 & $50(37.3 \%)$ \\
\hline N2 & $35(26.1 \%)$ \\
\hline \multicolumn{2}{|l|}{ Concurrent chemotherapy } \\
\hline No & $48(35.8 \%)$ \\
\hline Yes & $86(64.2 \%)$ \\
\hline
\end{tabular}

*TNM staging was determined based on the Clinical non operative treatment of esophageal cancer staging criteria (draft, 2010)

including only the GTV with reduced safety margins (PTV boost).

\section{Chemotherapy}

Patients with stage $\mathrm{T} 3$ and/or $\mathrm{N}+$ received concurrent cisplatin-based chemotherapy starting on the day before radiotherapy. Chemotherapy regimens were as follows:

Cisplatin $25 \mathrm{mg} / \mathrm{m}^{2}$, on days (d) $1-3$

Paclitaxel $135 \mathrm{mg} / \mathrm{m}^{2}(\mathrm{~d} 1)$ or5-FU $500 \mathrm{mg} / \mathrm{m}^{2}$, continuous on 5 days (d1-d5)
Two cycles were given concomitant to radiotherapy with 28 days between cycles

\section{F-FDG pet/CT}

18F-FDG PET/CT was performed at the following time points: 1)within 4 weeks before start of radiotherapy and chemotherapy (whole-body imaging) and 2)when 40-50 Gy to the PTV had been delivered (chest imaging, including all initial EC lesions). Metabolic parameters SUVmax, MTV, and TLG were measured. $\mathrm{SUV}_{\max } 1$, MTV1, and TLG1 indicates pretreatment values; $\mathrm{SUV}_{\max } 2, \mathrm{MTV} 2$, and TLG2 indicates values obtained during interim PET; $\triangle \mathrm{SUV}_{\max }, \triangle \mathrm{MTV}$, and $\triangle \mathrm{TLG}$ indicates the change (\%): $\triangle \mathrm{SUV}_{\max }=\left(\mathrm{SUV}_{\max } 1-\mathrm{SUV}\right.$ $\left.\max _{2} 2\right) / \mathrm{SUV}_{\max } 1, \quad \Delta \mathrm{MTV}=(\mathrm{MTV} 1-\mathrm{MTV} 2) / \mathrm{MTV} 1$, and $\triangle \mathrm{TLG}=(\mathrm{TLG} 1-\mathrm{TLG} 2) / \mathrm{TLG} 1$.

FDG PET/CT image acquisition was performed on a Discovery STE (GE Medical Systems, Milwaukee, WI, USA) with standard settings. 18F-FDG $(0.15 \mathrm{mCi} / \mathrm{kg})$ was generated using a cyclotron (Discovery STE; GE Medical Systems, Milwaukee, WI, USA) at our hospital. After injection, the patient rested for 50-60 min before the acquisition started and a 16-slice spiral CT scan was performed at $140 \mathrm{kV}, 120 \mathrm{mAs}, 3.75 \mathrm{~mm}$ thickness, and a $3.75 \mathrm{~mm}$ interval for attenuation correction calculation and anatomical localization.

Imaging data were interpreted by at least two experienced nuclear medicine physicians and, with reference to clinical signs and symptoms, gastroscopy, barium swallow testing, and CT images, were used to select the region of interest (ROI) in the esophageal lesions and the localized high radioactivity areas in the cervical, thorax, and abdominal areas at the Xeleris Workstation (GE Healthcare, Version 4.3). The selected ROI was manually adjusted to exclude physiologically high uptake areas such as the heart, and $40 \%$ of $\mathrm{SUV}_{\max }$ was used as the lower threshold for MTV calculations. SUV $\max$, mean SUV (mean of standard uptake value, $\mathrm{SUV}_{\text {mean }}$ ), MTV, and TLG were recorded during both 18F-FDG PET/CT scans. The parameters were calculated as follows: $\mathrm{SUV}=$ radioactivity of the sensitive area/ratio of the injected dose to the patient's weight, $\mathrm{SUV}_{\max }$ was the maximum SUV in the ROI, MTV was the volume included in the curve greater than or equal to $40 \% \mathrm{SUV}_{\text {max }}, \mathrm{SUV}_{\text {mean }}$ was the mean SUV in the MTV, and TLG was calculated as $\mathrm{SUV}_{\text {mean }} \times \mathrm{MTV}$. If patients did not have complete remission during (chemo-)radiotherapy, both PET scans would be co-registered and the parameters were determined based on the primary lesion's original location identified by visual method.

\section{Follow-up}

Patients were followed up from the start of treatment to the end of the follow-up period. Data from patients who died of any reasons were classified as complete data; 
data from patients who achieved tumor-free survival, or survival with tumor were censored at the date of the last follow-up information. Survival was defined as the time from the start of treatment to death or the end of follow-up. Barium swallow tests and chest 18F-FDG $\mathrm{PET} / \mathrm{CT}$ scan were performed one month after radiotherapy, then barium swallow tests and chest CT were performed every three months during the first 2 years and every six months for the next 3 years. After 5 years of treatment, barium swallow tests and chest CT scans were carried out once a year. Recurrence or metastasis needed to be confirmed by continuous imaging or biopsy.

\section{Statistical analysis}

Measurement data were expressed as the mean \pm standard deviation $(\mathrm{x} \pm \mathrm{s}$ ).SPSS Statics 22 (IBM, Armonk, NY) was used for all statistical analysis. Statistically significant parameters were analyzed using the operator characteristic curve (ROC) to obtain the optimal threshold based on the Youden index. The associations between endpoints and clinically relevant parameters (gender, age, tumour grade, $\mathrm{T}$ stage, $\mathrm{N}$ stage, UICC stage and localization) as well as quantitative PET parameters were analyzed using univariate Cox proportional hazards regression in which the PET parameters were included as binarized parameters. The Kaplan-Meier method (log-rank test) and Cox proportional hazards regression models were used to analyze OS and associated risk factors. Values $\leq \alpha=0.05$ were deemed statistically significant.

\section{Results}

\section{Clinical features, follow-up, and survival}

A total of 134 patients were eligible and included in this analysis. Patients were treated between July 2009 and October 2013. Follow up ended on October 1, 2017, with a median follow-up time of 62(47-99) months. 101 patients died and 33 patients survived during follow-up. The 1-, 3-, and 5-year overall survival rates were 66.4, 35.7 , and $24.5 \%$, respectively. The $1-, 3-$, and 5-year local control rates were $73.3,57.5$, and $54.5 \%$, respectively, and the 1-, 3-, and 5-year progress free rates were 48.5, 24.4 , and $21.3 \%$, respectively.

\section{Relationships between each baseline 18F-FDG PET/CT parameters and OS}

Mean $\mathrm{SUV}_{\max } 1$ was $13.9 \pm 6.0$, mean MTV1 was $18.8 \pm$ $18.8 \mathrm{~mL}$, and mean TLG1 was $159.4 \pm 154.0$. Univariate survival analysis showed that $\mathrm{SUV}_{\max } 1$ was not associated with OS (Table 2). As shown in Table 2 MTV1 and TLG1 were both associated with OS. The optimal prognostic threshold for OS (per the ROC and Youden index) were $10.5 \mathrm{~mL}$ for MTV1 and 59.8 for TLG1. Figure 1a and Fig. 1b show Kaplan-Meier plots for both parameters.. Log-Rank test of Kaplan-Meier survival analysis showed that
MTV1 $<10.5 \mathrm{~mL}$ and TLG1 $<59.8$ were associated with improved OS, with an estimated median survival of 36.9 months (95\% CI: 26.4-47.5, $p<0.0001$ ) and 48.9 months (95\% CI: 25-72.7, p $<0.0001)$, respectively.

\section{Relationships between interim 18F-FDG PET/CT parameters and OS}

Performed after application of $40 \mathrm{~Gy}$, the mean $\mathrm{SUV}_{\max } 2$ was $6.42 \pm 2.7$, the mean MTV2 was $13.9 \pm 11.0 \mathrm{~mL}$, and the mean TLG2 was $45.6 \pm 30.8$. Univariate survival analysis showed that MTV2 was not significantly associated with OS, while $\mathrm{SUV}_{\max } 2$ and TLG2 were significantly related to OS (Table 2).As show in Fig. 1c and Fig. 1d, the optimal prognostic threshold for OS was 7.8 for $\mathrm{SUV}_{\max } 2$ and 44.3 for TLG2. Log-Rank test of Kaplan-Meier survival analysis showed that $\mathrm{SUV}_{\max } 2<7.8$ and TLG $2<44.3$ were associated with improved OS, with an estimated median survival of 27.9 months $(p<0.001)$ and 32.6 months $(p<0.001)$, respectively.

\section{Relationships between changes in 18F-FDG PET/CT metabolic parameters and OS}

Mean $\triangle \mathrm{SUV}_{\max }$ was $0.46 \pm 0.34$, mean $\triangle \mathrm{MTV}$ was $0.25 \pm 1.42$, and mean $\Delta$ TLG was $0.38 \pm 0.92$.Univariate

Table 2 Univariate Cox regression analysis with respect to overall survival

\begin{tabular}{|c|c|c|c|c|}
\hline Parameter & Risk & $\mathrm{HR}$ & $95 \% \mathrm{Cl}$ & $p$ value \\
\hline \multicolumn{5}{|l|}{ Clinical parameters } \\
\hline Gender & Male & 1.18 & $0.74-1.89$ & 0.477 \\
\hline Age & $>58.5$ & 1 & $0.98-1.02$ & 0.698 \\
\hline Tumor_grade & $>2$ & 2.63 & $1.71-4.05$ & 0 \\
\hline T stage & $>3$ & 1.52 & $1.10-2.08$ & 0.01 \\
\hline N stage & $>1$ & 1.46 & $1.13-1.89$ & 0.003 \\
\hline UICC stage & $>\|$ & 2.19 & $1.50-3.22$ & 0 \\
\hline Localization & middle & 1.31 & $1.04-1.65$ & 0.02 \\
\hline Length of tumor & $>3.5$ & 1.18 & $1.08-1.28$ & 0 \\
\hline Concurrent chemo & No & 0.99 & $0.66-1.48$ & 0.943 \\
\hline \multicolumn{5}{|l|}{ PET parameter } \\
\hline SUV $V_{\max } 1$ & $>9.6$ & 1.01 & $0.98-1.04$ & 0.419 \\
\hline SUV $\max ^{2}$ & $>7.8$ & 1.16 & $1.07-1.25$ & 0 \\
\hline$\Delta S U V_{\max }$ & $<0.23$ & 0.85 & $0.51-1.42$ & 0.538 \\
\hline MTV1 & $>10.5$ & 1.02 & $1.01-1.03$ & 0 \\
\hline MTV2 & $>15.9$ & 1.01 & $0.99-1.03$ & 0.395 \\
\hline$\Delta \mathrm{MTV}$ & $>0.075$ & 1.17 & $0.98-1.40$ & 0.001 \\
\hline TLG1 & $>59.8$ & 1 & $1.0-1.01$ & 0.001 \\
\hline TLG2 & $>44.3$ & 1.01 & $1.0-1.01$ & 0.019 \\
\hline$\Delta T L G$ & $>0.27$ & 1.21 & $0.91-1.62$ & 0.014 \\
\hline
\end{tabular}

HR Hazard ratio, Cl Confidence interval

Values in bold are showing at least trend for significance with $p<0.1$ in univariate analyses 


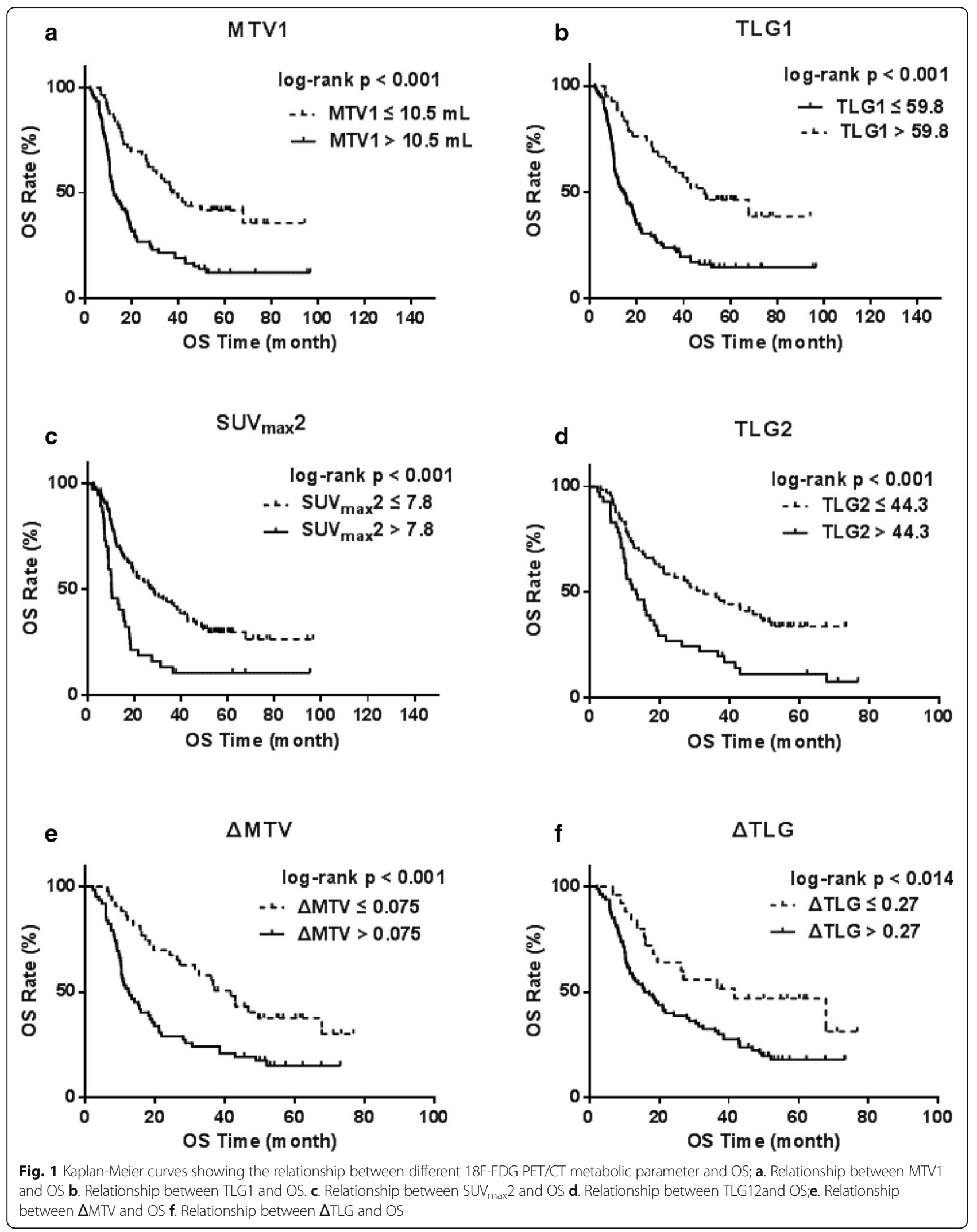


survival analysis showed that $\Delta \mathrm{SUV}_{\text {max }}$ was not significantly associated with OS, while $\triangle \mathrm{MTV}$ and $\triangle \mathrm{TLG}$ both showed a significant association with OS (Table 2). The optimal prognostic thresholds for OS (per the ROC and Youden index) were 0.075 and 0.27 , respectively. Kaplan-Meier survival analysis showed that $\Delta \mathrm{MTV}<0.075$ and $\triangle \mathrm{TLG}<0.27$ were associated with improved OS, with an estimated median survival of 41.6 months for both parameters $(\mathrm{p}<0.001, p<0.014)$ (Fig. 1e and Fig. 1f).

\section{Relationships between clinical features and OS}

Univariate survival analysis showed that UICC stage and lesion length were significantly associated with OS. ROC analysis showed that Stage I-II and a lesion length (Length_T) $\leq 3.5 \mathrm{~cm}$ was associated with improved median survival, which was 67.8 months $(p<0.001)$ in stage I-II patients and was not reached in the group with a tumor length_T $\leq 3.5 \mathrm{~cm}(\mathrm{p}<0.001)$, respectively (Fig. 2$)$.

\section{Multivariate analysis}

UICC stage, Length_T, SUV $\max _{2} 2$, MTV1, $\Delta$ MTV, TLG1, TLG2, and $\triangle$ TLG were incorporated into Cox multivariate regression analysis; the results showed that UICC stage, length_T, SUV $\mathrm{max}_{2}$, MTV1 and TLG2 were independent prognostic factors for OS $(p<0.05)$, with Wald values of 9.8,5.5,9.77,4.82 and 10.9 and hazard ratios of 2.7,2.1, 2,1.7 and 2.2, respectively (see detail in Table 3).

\section{Discussion}

Radiotherapy and chemotherapy are important treatment approaches for EC [21]. For non-surgical EC treatment, radiotherapy with concurrent chemotherapy is the treatment of choice. Anatomical information from conventional barium swallow tests or CT cannot reflect the burden of metabolic active tumor volume. 18F-FDG PET/CT functional imaging was shown to be superior to conventional imaging for EC staging [22, 23]. A growing body of evidence shows that 18F-FDG PET/CT is a valuable tool with independent prognostic information for EC patients $[24,25]$. However, studies about sequential 18F-FDG $\mathrm{PET} / \mathrm{CT}$ in predicting (chemo-)radiation outcomes for EC are relatively sparse, especially in China. This study shows that TLG and its percentage change during radiotherapy have prognostic value regarding OS of EC patients. The same holds true for MTV. However, in our study baseline $\mathrm{SUV}_{\max }$ did not have any prognostiv value. Interim SUVmax2 after about 40Gy of radiotherapy was at the other hand significantly associated with OS. These results suggest that in terms of prognostic stability, TLG and MTV seem to be quite robust in this cohort of patients.

$\mathrm{SUV}_{\max }$ is commonly used as a prognostic parameter in clinical practice as it is easy to measure. However, $\mathrm{SUV}_{\max }$ is affected by acquisition intervals, blood glucose, and insulin levels. In addition, $\mathrm{SUV}_{\max }$ focuses on a single voxel with the highest FDG uptake, and thus cannot be used to evaluate the tumor's overall metabolic state. As a result, researchers continue to debate the prognostic value of pretreatment $\mathrm{SUV}_{\max }[12-15]$. That may be the reason why our study showed that baseline $\mathrm{SUV}_{\max } 1$ was not associated with OS. SUV $\max _{2} 2$ was significantly associated with OS. This could be because $\mathrm{SUV}_{\max }$ during treatment reflects the maximum metabolic activity of tumor tissue after antitumor treatment, e.g. response to therapy. Thus, a high value indicates high metabolic activity after conventional radiotherapy and chemotherapy. This non-response seems to be associated with high risk of local relapse and distant metastasis, and therefore with a worse prognosis. This finding is in line with one study that investigated interim PET and pathological examination of EC specimens' response after neoadjuvant therapy [26].

MTV is determined based on a SUV threshold and ideally should reflect metabolically active tumor cells with increased glucose metabolism; thus, it reflects the overall metabolic state of the tumor. Several studies showed that MTV is a better prognostic parameter than $\mathrm{SUV}_{\max }[20,27-29]$. This study showed that both baseline MTV1 and $\triangle \mathrm{MTV}$ were correlated with OS. ROC curve analysis showed that the optimal prognostic threshold for OS was $10.5 \mathrm{~mL}$ for MTV1. The published MTV thresholds varied across studies; nevertheless, low baseline MTV indicates a low tumor burden and is associated with a more favorable prognosis. Theoretically,
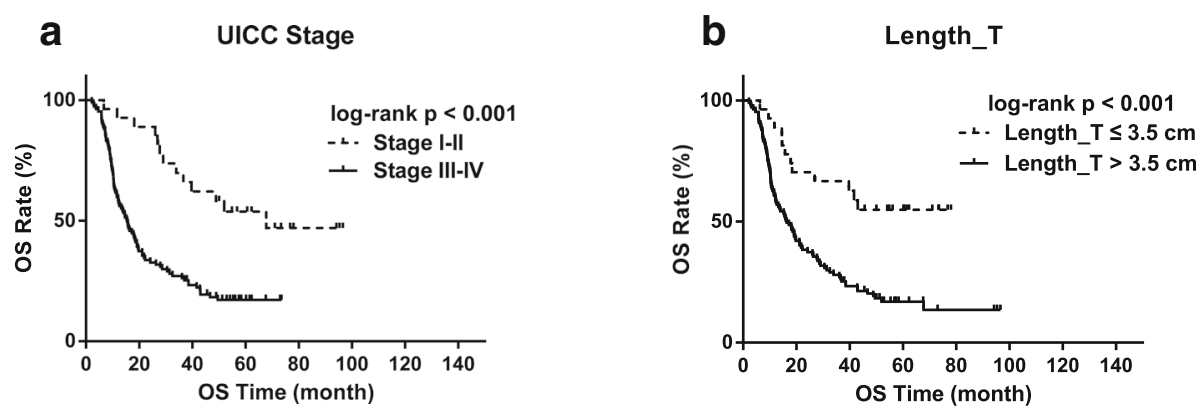

Fig. 2 Kaplan-Meier curves for overall survival (OS) in patients stratified by UICC stage and length of primary tumor 
Table 3 Cox regression results

\begin{tabular}{|c|c|c|c|c|c|c|c|c|c|}
\hline & & UICC Stage & Length_T & SUV max $^{2}$ & MTV1 & $\triangle \mathrm{MTV}$ & TLG1 & TLG2 & $\triangle \mathrm{TLG}$ \\
\hline Wald & & 9.8 & 5.5 & 9.77 & 4.82 & 2.72 & 2.11 & 10.9 & 0.09 \\
\hline$P$ valne & & 0.002 & 0.019 & 0.002 & 0.028 & 0.099 & 0.146 & 0.010 & 0.770 \\
\hline$H R$ & & 2.7 & 2.1 & 2 & 1.7 & 1.53 & 0.648 & 2.2 & 0.9 \\
\hline \multirow[t]{2}{*}{$95 \% \mathrm{Cl}$} & Upper limit & 1.5 & 1.13 & 1.3 & 1.1 & 0.9 & 0.36 & 1.4 & 0.44 \\
\hline & Lower limit & 5.2 & 3.9 & 3.1 & 2.8 & 2.5 & 1.16 & 3.5 & 1.84 \\
\hline
\end{tabular}

HR Hazard ratio, Cl Confidence interval

MTV during radiotherapy reflects the volume of radiotherapy-resistant tumor cells; however, this study was not able to show that MTV2 was associated with OS. This may be because some patients had radiation induced inflammation in the tissue around the esophageal tumor after radiotherapy and chemotherapy, which interfered with the MTV measurement. As a result, MTV2 may not be able to properly reflect the actual interim-PET MTV, which was consistent with the findings from Erasmus et al. [16], Bar-Ad et al. [17], and Yuan et al [18]

TLG has recently become an emerging new prognostic 18F-FDG PET/CT parameter. TLG is the product of MTV and SUV mean. Studies have shown that TLG is non-inferior [30-32] and may even be superior to MTV $[19,20,33]$ as a predictor, probably because it reflects both tumor metabolic activity and tumor volume. This study showed that all three TLG parameters, TLG1, TLG2, and $\triangle \mathrm{TLG}$, were related to OS $(p<0.05)$, suggesting that TLG is more robust and reliably to predict the outcome of radical (chemo-)radiotherapy in EC. However upon multivariate regression testing TLG1 and $\triangle T L G$ did not remain independent prognostic factors, which might however be due to the correlation with MTV and other PET parameters.

At present, $\mathrm{SUV}_{\text {max }}$, MTV, and TLG are commonly used $\mathrm{PET} / \mathrm{CT}$ parameters to predict tumor response in interim PET. This study showed that TLG was a reliable sequential 18F-FDG PET/CT parameter for predicting outcome after radical (chemo-)radiation for EC. TLG could be less prone to the shortcomings of $\mathrm{SUV}_{\max }$ and MTV discussed above. However it is important to mention that the calculation of TLG is strongly dependent on tumor delineation. Therefore also TLG has several limitations, even more when used during interim PET (TLG2 or $\triangle \mathrm{TLG}$ ) since there is no consensus for optimal delineation of FDG metabolic tumors in the course of radiotherapy. Simultaneously, like MTV, TLG also has the drawbacks of being influenced by inflammation induced by (Chemo-)radiation. That may be another reason why the Cox multi-factor regression did not show positive results for $\triangle T L G$ in our study. Nontumour-affected oesophagus (NTO) on restaging PET may be a promising method to deal with the treatment-induced inflammation [34]. Additional PET biomarkers are under investigation for EC right now. Recent studies [35, 36] showed that texture analysis may become a promising new method for predicting EC prognosis; however, texture analysis is difficult to perform in clinical practice, and the results vary greatly across software packages. Furthermore, new tracers may minimize interference from radiation inflammation on PET/CT images [37].

The results of our research show that interim PET after $40 \mathrm{~Gy}$ of radiotherapy seems to be a promising tool to comprehensively evaluate the treatment response with reference to the baseline parameters. $\mathrm{SUV}_{\max } 2$ and TLG2 seem to be useful parameters to identify patients with high risk of recurrence. Patients with adverse prognostic factors may receive re-planning and higher dose to the region of high FDG uptake or additional therapeutic escalation to improve treatment outcomes or even might undergo surgery.

This study has some limitations. First, no official MTV threshold has been established, and we used the volume of the area greater than $40 \% \mathrm{SUV}_{\max }$, which requires further validation. Second, the heterogeneity in concurrent chemotherapy regimens may affect the results. Third, results can be prone to bias, inherent to any retrospective evaluation and should be interpreted as hypothesis generating.

\section{Conclusion}

The results of our study indicate that sequential 18F-FDG PET/CT metabolic parameters have a good prognostic value for OS of squamous EC. It is recommended to repeat 18F-FDG PET/CT scan during chemoradiotherapy to improve clinical decision-making and individualize treatment.

\section{Abbreviations \\ Cl: Confidence interval.; CT: Computed tomography; CTV: Clinical target volume; EC: Esophageal cancer; FDG: Fluorodeoxyglucose; GTV: Gross tumor volume; Gy: Gray; HR: Hazard ratio; ICRU: International Commission on Radiation Units and Measurements; KPS: Karnofsky performance status; m: Months; MTV: Metabolic tumor volume; NTO: Non-tumour-affected oesophagus; OS: Overall survival; PET: Positron emission tomography; PTV: Planning target volume; ROC: Receiver operating characteristic curve; ROI: Region of interest; SUV: Standard uptake value; TLG: Total lesion glycolysis}

\section{Acknowledgements}

Not applicable.

Funding

This work was partly supported by the Xiamen Science and Technology Program, (3502Z20164009) and Scientific Research Foundation for Youth 
Talent of Xiamen Cancer Hospital, First Affiliated Hospital of Xiamen University (ZLYYB201801).

\section{Availability of data and materials}

The present data is summarized in this paper. The complete dataset can be retrieved from the authors upon formal request from interested readers.

\section{Authors' contributions}

HW provided ideas for the study. YL, SZ, and QL performed the analysis and drafted the manuscript. SC designed the figures and calculated the underlying statistics. YL, QL, LLC and HW were responsible for treatment, imaging, collection of patient data and follow up. QL, HW, SZ and YL provided ideas, supervised the analysis and interpretation of the data and reviewed the manuscript. All authors read and approved the final manuscript.

\section{Ethics approval and consent to participate}

All patients gave their written informed consent. All data were retrospectively analyzed in an anonymized form.

\section{Consent for publication}

Not applicable.

\section{Competing interests}

The authors declare that they have no competing interests.

\section{Publisher's Note}

Springer Nature remains neutral with regard to jurisdictional claims in published maps and institutional affiliations.

\section{Author details}

'Department of Radiation Oncology, Xiamen Cancer Hospital, the First Affiliated Hospital of Xiamen University, Xiamen, China. ${ }^{2}$ Department of Radiation Oncology, Charité Universitätsmedizin Berlin, Berlin, Germany. ${ }^{3}$ Department of Nuclear Medicine, Xiamen Cancer Hospital, the First Affiliated Hospital of Xiamen University/Southern Fujian PET Center, Xiamen, China.

\section{Received: 19 September 2018 Accepted: 5 February 2019}

\section{Published online: 19 February 2019}

\section{References}

1. Jemal A, Bray F, Center MM, et al. Global cancer statistics [J]. CA Cancer J Clin. 2011;61(2):69-90.

2. Gu X, Yin W, Yu Z, et al. Radiation oncology. 4rd ed. In: Peking union medical college press; 2008.

3. Shahbaz Sarwar CM, Luketich JD, Landreneau RJ, et al. Esophageal cancer: an update[J]. Int J Surg. 2010;8(6):417-22.

4. He J. Chinese Cancer society professional Committee for Esophageal Cancer, guidelines for the standardized diagnosis and treatment of esophageal Cancer (second edition)[S]. Beijing: China Union Medical College Press; 2013. p. 99-107.

5. Naik KB, Liu Y, Goodman M, et al. Concurrent chemoradiotherapy with or without surgery for patients with resectable esophageal cancer: an analysis of the National Cancer Data Base. Cancer. 2017:123:3476-85.

6. Kranzfelder M, Schuster T, Geinitz H, et al. Metaanalysis of neoadjuvant treatment modalities and definitive nonsurgical therapy for oesophageal squamous cell cancer. Br J Surg. 2011;98:768-83.

7. Yang $\mathrm{H}$, Liu H, Chen $\mathrm{Y}$, et al. Neoadjuvant Chemoradiotherapy followed by surgery versus surgery alone for locally advanced squamous cell carcinoma of the esophagus (NEOCRTEC5010): a phase III multicenter, randomized, open-label clinical trial. J Clin Oncol. 2018;36(27):2796-803.

8. van Hagen P, Hulshof MCCM, van Lanschot JJB, et al. Preoperative chemoradiotherapy for esophageal or junctional cancer. N Engl J Med. 2012:366:2074-84

9. Shum WY, Ding HJ, Liang JA, et al. Use of pretreatment metabolic tumor volumes on PET-CT to predict the survival of patients with squamous cell carcinoma of esophagus treated by curative surgery[J]. Anticancer Res. 2012;32(9):4163-8

10. Sun G, Tian JM, Gorospe EC, et al. Utility of baseline positron emission tomography with computed tomography for predicting endoscopic resectability and survival outcomes in patients with early esophageal adenocarcinoma [J]. J Gastroenterol Hepatol. 2013;28(6):975-81.
11. Wieder HA, Ott K, Lordick F, et al. Prediction of tumor response by FDG-PET: comparison of the accuracy of single and sequential studies in patients with adenocarcinomas of the esophagogastric junction [J]. Eur J Nucl Med Mol Imaging. 2007:34(12):1925-32.

12. Omloo JM, Heijl MV, Hoekstra OS, et al. FDG-PET parameters as prognostic factor in esophageal cancer patients: a review[J]. Ann Surg Oncol. 2011;18(12):3338-52.

13. Wiele CV, Kruse V, Smeets $P$, et al. Predictive and prognostic value of metabolic tumour volume and total lesion glycolysis in solid tumours[J]. Eur J Nucl Med Mol Imaging. 2013;40(2):290-301.

14. Hatt M, Visvikis D, Albarghach NM, et al. Prognostic value of 18F-FDG PET image-based parameters in oesophageal cancer and impact of tumour delineation methodology[J]. Eur J Nucl Med Mol Imaging. 2011;38(7):1191-202.

15. Jayachandran P, Pai RK, Quon A, et al. Postchemoradiotherapy positron emission tomography predicts pathologic response and survival in patients with esophageal cancer[J]. Int J Radiat Oncol Biol Phys. 2012; 84(2):471-7.

16. Erasmus JJ, Munden RF, Truong MT, et al. Preoperative chemo-radiationinduced ulceration in patients with esophageal cancer: a confounding factor in tumor response assessment in integrated computed tomographic-positron emission tomographic imaging[J]. J Thorac Oncol. 2006;1(5):478-86

17. Barad V, Leiby B, Witek M, et al. Treatment-related acute esophagitis for patients with Locoregionally advanced non-small cell lung Cancer treated with involved-field radiotherapy and concurrent chemotherapy[J]. Am J Clin Oncol. 2014;37(5):433-7.

18. Yuan ST, Brown RK, Zhao L, et al. Timing and intensity of changes in FDG uptake with symptomatic esophagitis during radiotherapy or chemo-radiotherapy[J]. Radiat Oncol. 2014;9(1):37.

19. Chang S, Koo PJ, Kwak JJ, et al. Changes in Total lesion glycolysis evaluated by repeated F-18 FDG PET/CT as prognostic factor in locally advanced esophageal Cancer patients treated with preoperative Chemoradiotherapy[J]. Oncology. 2016;90(2):97-102.

20. Hong $\mathrm{JH}$, Kim HH, Han EJ, et al. Total lesion glycolysis using ${ }^{18} \mathrm{~F}$-FDG PET/CT as a prognostic factor for locally advanced esophageal Cancer[J]. J Korean Med Sci. 2016:31(1):39-46.

21. Chinese non surgical treatment of clinical staging of esophageal cancer expert group. Clinical non operative treatment of esophageal cancer staging criteria (draft) [J]. Chin. J Radiat Oncol. 2010;19:179-80.

22. Cools-Lartigue SJ, Ferri LE. Current status of management of malignant disease: current management of esophageal cancer[J]. J Gastrointest Surg. 2015:19(5):964-72.

23. Yuan SH, Yu JM, Yu YH, et al. FDG PET/CT versus PET alone for pre-surgica detection of lymph node metastasis in esophageal carcinoma[J]. Chinese Journal of Oncology. 2007:29(3):221-4.

24. Calais J, Dubray B, Nkhali L, et al. High FDG uptake areas on preradiotherapy $\mathrm{PET} / \mathrm{CT}$ identify preferential sites of local relapse after chemoradiotherapy for locally advanced oesophageal cancer[J]. Eur J Nuc Med Mol Imaging. 2015:42(6):858-67.

25. Yuan $\mathrm{H}$, Tong DK, Vardhanabhuti $\mathrm{V}$, et al. PET/CT in the evaluation of treatment response to neoadjuvant chemoradiotherapy and prognostication in patients with locally advanced esophageal squamous cell carcinoma[J]. Nucl Med Commun. 2016:37(9):947-55.

26. Higuchi I, Yasuda T, Yano M, et al. Lack of fludeoxyglucose F 18 uptake in posttreatment positron emission tomography as a significant predictor of survival after subsequent surgery in multimodality treatment for patients with locally advanced esophageal squamous cell carcinoma[J]. J Thorac Cardiovasc Surg, 2008, 136(1): 205-212, 212 e1-3.

27. Li Y, Lin Q, Zhao L, et al. Pre-treatment metabolic tumor volume and total lesion glycolysis are useful prognostic factors for esophageal squamous cell cancer patients[J]. Asian Pac J Cancer Prev. 2014;15(3):1369-73.

28. Soydal Ç, Yüksel C, KüçükNÖ, et al. prognostic value of metabolic tumor volume measured by 18F-FDG PET/CT in esophageal Cancer patients[J]. Mol Imaging Radionucl Ther, 2014, 23(1): 12.

29. Zhu WQ, Yu JM, Sun XR, et al. The prognostic value of metabolic tumor volume in FDG PET/CT evaluation of post-operative survival in patients with esophageal squamous cell cancer[J]. Chinese. J Nucl Med. 2011;31(6):378-81.

30. Olivier A, Petyt G, Cortot A, et al. Higher predictive value of tumour and node [18F]-FDG PET metabolic volume and TLG in advanced lung cancer under chemotherapy[J]. Nucl Med Commun. 2014;35(9):908-15. 
31. Dibble EH, Alvarez AC, Truong MT, et al. 18F-FDG metabolic tumor volume and total glycolytic activity of oral cavity and oropharyngeal squamous cell cancer: adding value to clinical staging[J]. J Nucl Med. 2012;53(5):709-15.

32. Lee JW, Kang $\mathrm{CM}$, Choi $\mathrm{HJ}$, et al. Prognostic value of metabolic tumor volume and Total lesion glycolysis on preoperative 18F-FDG PET/CT in patients with pancreatic Cancer[J]. J Nucl Med. 2014;55(6):898-904.

33. Li YM, Lin Q, Luo ZM, et al. Value of sequential 18F-fluorodeoxyglucose positron emission tomography/computed tomography (FDG PET/CT) in prediction of the overall survival of esophageal cancer patients treated with chemoradiotherapy [J]. Int J Clin Exp Med. 2015;8(7):10947-55.

34. Li Y, Hofheinz F, Furth C, et al. Increased evidence for the prognostic value of FDG uptake on late-treatment PET in non-tumour-affected oesophagus in irradiated patients with oesophageal carcinoma.[J]. Eur J Nucl Med Mol Imaging. 2018;45(10):1752-61.

35. Dong $X$, Xing L, Wu P, et al. Three-dimensional positron emission tomography image texture analysis of esophageal squamous cell carcinoma: relationship between tumor 18F-fluorodeoxyglucose uptake heterogeneity, maximum standardized uptake value, and tumor stage[J]. Nucl Med Commun. 2013;34(1):40-6.

36. Ganeshan B, Skogen K, Pressney I, et al. Tumour heterogeneity in oesophageal cancer assessed by $\mathrm{CT}$ texture analysis: preliminary evidence of an association with tumour metabolism, stage, and survival[J]. Clin Radiol. 2012;67(2):157-64.

37. Chen H, Li Y, Wu H, et al. 3'-deoxy-3'-[18F]-fluorothymidine PET/CT in early determination of prognosis in patients with esophageal squamous cell cancer: comparison with [18F]-FDG PET/CT[J]. Strahlenther Onkol. 2015;191(2):141-52.

Ready to submit your research? Choose BMC and benefit from:

- fast, convenient online submission

- thorough peer review by experienced researchers in your field

- rapid publication on acceptance

- support for research data, including large and complex data types

- gold Open Access which fosters wider collaboration and increased citations

- maximum visibility for your research: over $100 \mathrm{M}$ website views per year

At $\mathrm{BMC}$, research is always in progress.

Learn more biomedcentral.com/submissions 